\title{
Mixed reality for robotic treatment of a splenic artery aneurysm
}

\author{
Andrea Pietrabissa $\cdot$ Luca Morelli $\cdot$ Mauro Ferrari · \\ Andrea Peri · Vincenzo Ferrari · Andrea Moglia · \\ Luigi Pugliese $\cdot$ Fabio Guarracino $\cdot$ Franco Mosca
}

Received: 9 June 2009/Accepted: 26 August 2009/Published online: 14 October 2009

(C) Springer Science+Business Media, LLC 2009

\begin{abstract}
Background Techniques of mixed reality can successfully be used in preoperative planning of laparoscopic and robotic procedures and to guide surgical dissection and enhance its accuracy.

Methods A computer-generated three-dimensional (3D) model of the vascular anatomy of the spleen was obtained from the computed tomography (CT) dataset of a patient with a $3-\mathrm{cm}$ splenic artery aneurysm. Using an environmental infrared localizer and a stereoscopic helmet, the surgeon can see the patient's anatomy in transparency (augmented or mixed reality). This arrangement simplifies correct positioning of trocars and locates surgical dissection directly on top of the aneurysm. In this way the
\end{abstract}

Electronic supplementary material The online version of this article (doi:10.1007/s00464-009-0703-0) contains supplementary material, which is available to authorized users.

A. Pietrabissa $(\varangle) \cdot$ L. Morelli · A. Peri · L. Pugliese ·

F. Mosca

Sezione di Chirurgia Mininvasiva, Divisione di Chirurgia I

Universitaria, Dipartimento di Oncologia, dei Trapianti e Delle

Nuove Tecnologie in Medicina, Università di Pisa,

Ospedale di Cisanello, via Paradisa 2, 56124 Pisa, Italy

e-mail: apietrab@med.unipi.it

M. Ferrari

Divisione di Chirurgia Vascolare, Dipartimento di Oncologia, dei Trapianti e Delle Nuove Tecnologie in Medicina,

Università di Pisa, Pisa, Italy

V. Ferrari · A. Moglia

Center for Computer Assisted Surgery ENDOCAS,

Università di Pisa, Pisa, Italy

F. Guarracino

Divisione di Anestesiologia,

Azienda Ospedaliero-Universitaria Pisana, Pisana, Italy surgeon limits unnecessary dissection, leaving intact the blood supply from the short gastric vessels and other collaterals. Based on preoperative planning, we were able to anticipate that the vascular exclusion of the aneurysm would result in partial splenic ischemia. To re-establish the flow to the spleen, end-to-end robotic anastomosis of the splenic artery with the Da Vinci surgical system was then performed. Finally, the aneurysm was fenestrated to exclude arterial refilling.

Results The postoperative course was uneventful. A control CT scan 4 weeks after surgery showed a wellperfused and homogeneous splenic parenchyma. The final 3D model showed the fenestrated calcified aneurysm and patency of the re-anastomosed splenic artery.

Conclusions The described technique of robotic vascular exclusion of a splenic artery aneurysm, followed by reanastomosis of the vessel, clearly demonstrates how this technology can reduce the invasiveness of the procedure, obviating an otherwise necessary splenectomy. Also, the use of intraoperative mixed-reality technology proved very useful in this case and is expected to play an increasing role in the operating room of the future.

Keywords Vascular (blood vessels) · Human/robotic . Imaging $\cdot$ VR

Disclosures Drs. Andrea Pietrabissa, Luca Morelli, Mauro Ferrari, Andrea Peri, Vincenzo Ferrari, Andrea Moglia, Luigi Pugliese, Fabio Guarracino, and Franco Mosca have no conflicts of interest or financial ties to disclose. 